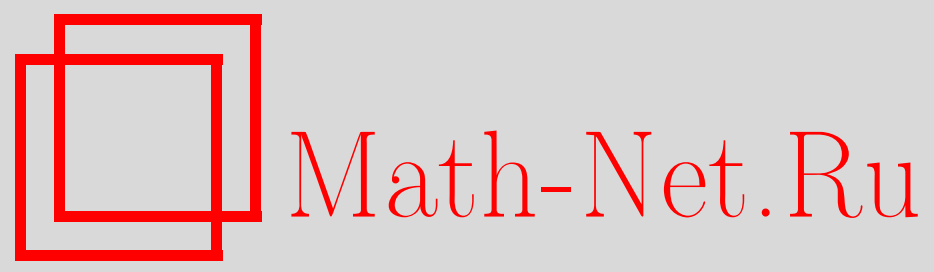

В. Г. Михайлов, Предельная теорема Пуассона для числа неколлинеарных решений системы случайных уравнений специального вида, Дискрет. матем., 2001, том 13, выпуск $3,81-90$

DOI: https://doi.org/10.4213/dm296

Использование Общероссийского математического портала Math-Net.Ru подразумевает, что вы прочитали и согласны с пользовательским соглашением http: //www.mathnet.ru/rus/agreement

Параметры загрузки:

IP: 54.198 .187 .58

26 апреля 2023 г., 18:03:25

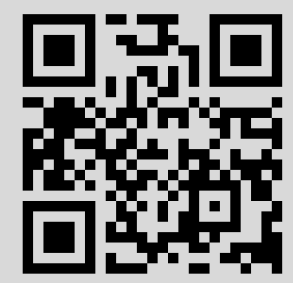




\title{
Предельная теорема Пуассона для числа неколлинеарных решений системы случайных уравнений специального вида
}

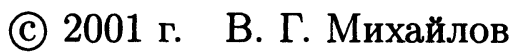

Исследуются свойства числа $\nu$ неколлинеарных ненулевых решений системы случайных уравнений следующего вида. Левые части уравнений записаны как функции от выражений вида

$$
l_{s}=a_{s, 1} x_{1} \oplus \ldots \oplus a_{s, n} x_{n}
$$

(сложение производится в поле $G F(q)$ ) со случайными коэффициентами при переменных, а правые части равны нулю. Предполагается, что коэффициенты в выражения $l_{s}$ распределены независимо и равновероятно. Система рассматривается относительно неизвестных $x_{1}, \ldots, x_{n} \in G F(q)$. Получены неравенства для факториальных моментов случайной величины $\nu$, а также достаточные условия выполнения для $\nu$ предельной теоремы Пуассона.

Работа выполнена при поддержке Российского фонда фундаментальных исследований, проект 99-01-00012, и Совета по грантам Президента РФ и государственной поддержке ведущих научных школ, проект 00-15-96136.

Пусть $q$ - простое число или $q=p^{d}$ - степень простого числа $p, f_{1}, \ldots, f_{T}-$ некоторые функции из множеств $G F(q)^{m_{1}}, \ldots, G F(q)^{m_{T}}$, соответственно, в $G F(q)$. Рассмотрим систему случайных уравнений над полем $G F(q)$

$$
f_{t}\left(l_{t}^{1}, \ldots, l_{t}^{m_{t}}\right)=0, \quad t=1, \ldots, T
$$

где

$$
l_{t}^{k}=a_{t, 1}^{k} x_{1} \oplus \ldots \oplus a_{t, n}^{k} x_{n}
$$

- линейные над $G F(q)$ функции от неизвестных $x_{1}, \ldots, x_{n}$. Здесь и далее символ $\oplus$ мы используем как для обозначения операции сложения в $G F(q)$, так и для обозначения операций сложения векторов в линейных пространствах над полями $G F(q)$ и $G F(p)$. Система (1)-(2) является системой из $T$ уравнений относительно неизвестных $x_{1}, \ldots, x_{n}$. Коэффициенты $a_{t, i}^{k}$ линейных функций $l_{t}^{k}$ предполагаются независимыми в совокупности случайными величинами, распределенными равномерно на $G F(q)$. Поэтому все уравнения в нашей системе являются случайными. Случайным является и число решений системы (1). Мы изучим число $\nu$ неколлинеарных ненулевых решений системы (1). 
Для функции $f$ от $m$ переменных введем обозначения

$$
B(f)=\left\{\left(z_{1}, \ldots, z_{m}\right): f\left(z_{1}, \ldots, z_{m}\right)=0\right\} .
$$

Положим

$$
\Lambda=\left(q^{n}-1\right) \prod_{t=1}^{T}\left(q^{-m_{t}}|B(f)|\right) .
$$

Здесь и далее $|B|$ обозначает число элементов конечного множества $B$. Можно показать (см. равенство (15)), что $\Lambda=E \nu$.

Заметим, что линейное пространство $V^{m}$ над полем $G F(q), q=p^{d}$, может рассматриваться как $m d$-мерное линейное пространство над полем $G F(p)$. Назсзем множество $B \subseteq V^{m} p$-аффинным над $G F(q)$, если найдутся такие

$$
k \leqslant m d, \quad c_{i, 1}, \ldots, c_{i, m d}, \quad b_{i} \in G F(p), \quad i=1, \ldots, k,
$$

при которых

$$
B=\left\{\left(u_{1}, \ldots, u_{m d}\right): c_{i, 1} u_{1} \oplus \ldots \oplus c_{i, m d} u_{m d}=b_{i}, \quad i=1, \ldots, k\right\} .
$$

Это определение означает, что если множество $B(f)$ является $p$-аффинным, то уравнение $f\left(z_{1}, \ldots, z_{m}\right)=0$ эквивалентно некоторой системе линейных уравнений над полем $G F(p)$.

Пусть $\chi(B)$ - индикатор того, что множество векторов $B \subseteq V^{m}$ не является $p$-аффинным. Пусть $(m)_{r}=m(m-1) \ldots(m-r+1)$.

Теорема 1. При $n \geqslant r \geqslant 1$ выполнены неравенства

$$
\begin{aligned}
& \mathbf{E}(\nu)_{r} \geqslant \Lambda^{r} \prod_{s=0}^{r-1} \frac{q^{n}-q^{s}}{q^{n}-1}, \\
& \mathbf{E}(\nu)_{r}<\Lambda^{r}+\left(\Lambda+q^{r-1}\right)^{r} \prod_{t=1}^{T}\left(1-\chi\left(B\left(f_{t}\right)\right)\left|B\left(f_{t}\right)\right|^{-2}\right) .
\end{aligned}
$$

Теорема 2. Пустъ параметры $n$ и $T$ стремятся $к$ бесконечности, а функции $f_{1}, \ldots, f_{T}$ меняяотся так, что $\Lambda \rightarrow \lambda, 0<\lambda<\infty, u$

$$
\sum_{t=1}^{T} \chi\left(B\left(f_{t}\right)\right)\left|B\left(f_{t}\right)\right|^{-2} \rightarrow \infty .
$$

Тогда при любом фиксированном $r=1,2, \ldots$

$$
\mathbf{E}(\nu)_{r} \rightarrow \lambda^{r},
$$

а распределение случайной величины $\nu$ сходитсл $x$ распределению Пуассона $с$ параметром $\lambda$.

Замечание 1. Пусть в теореме 2 функции $f_{1}, \ldots, f_{T}$ таковы, что

$$
\sum_{t=1}^{T} \chi\left(B\left(f_{t}\right)\right)\left|B\left(f_{t}\right)\right|^{-2} \geqslant \varphi(n, T) \rightarrow \infty .
$$

Тогда сходимость моментов и распределения случайной величины $\nu$ является равномерной по всем наборам $f_{1}, \ldots, f_{T}$, удовлетворяющим этим условиям. 
Теорема 3. Пусть параметры $n$ и $T$ стремлтсл $x$ бесконечности, а бункции $f_{1}, \ldots, f_{T}$ меняются так, что выполнены условия

$$
\Lambda \rightarrow \infty, \quad \Lambda \leqslant \frac{n}{10}, \quad \Lambda^{2} \leqslant \frac{1}{49 \ln 2} \sum_{t=1}^{T} \chi\left(B\left(f_{t}\right)\right)\left|B\left(f_{t}\right)\right|^{-2} .
$$

Тогда функция распределения случайной величины $(\nu-\Lambda) \Lambda^{-1 / 2}$ сходится $\propto$ стандартной нормалъной функции распределения.

Замечание 2. Случай

$$
q=2, \quad f_{1}=\ldots=f_{T}=\left(z_{1} \oplus a_{1}\right) \ldots\left(z_{m} \oplus a_{m}\right),
$$

где $a_{1}, \ldots, a_{T} \in G F(2)$, изучался в [1], где были получены утверждения, аналогичные теоремам 1-3, а также ряд иных результатов. В [2] эти результаты были распространены на системы (1) с булевыми функциями

$$
f_{t}=\left(z_{1} \oplus a_{1}\right) \ldots\left(z_{m_{t}} \oplus a_{m_{t}}\right), \quad t=1, \ldots, T .
$$

Кроме того, в [2] было показано, что в случае (9) условие (5) можно заменить существенно более слабым условием

$$
\sum_{t=1}^{T} \frac{2^{m_{t}}-2}{\left(2^{m_{t}}-1\right)^{2}} \rightarrow \infty
$$

которое оказывается не только достаточным, но и необходимым для сходимости моментов случайной величины $\nu$ к моментам предельного пуассоновского распределения. Заметим, что при $q=2$ все ненулевые векторы в $V^{n}$ неколлинеарны и величина $\nu$ равна числу ненулевых решений.

Замечание 3. Если к системе (1)-(2) применить невырожденную линейную замену переменных, то это не изменит совместного распределения случайных коэффициентов. Поэтому множество неколлинеарных ненулевых решений (обозначим его через $W$ ) имеет распределение, инвариантное относительно таких замен переменных. В частности,

$$
\mathbf{P}\{W=\{x\}|| W \mid=1\}=\frac{1}{q^{n}-1}, \quad x \in V^{n} \backslash\{0\}
$$

a

$$
\mathbf{P}\{W=\{x, y\}|| W \mid=2\}=\frac{2}{\left(q^{n}-1\right)\left(q^{n}-q\right)},
$$

где $x, y \in V^{n} \backslash\{0\}, x \neq y$.

Описание основных задач теории систем случайных уравнений над конечными полями можно найти в книгах $[3,4]$ и статьях $[5,6]$. Надо сказать, что для изученных классов систем случайных булевых уравнений число решений, как правило, имеет предельные распределения, отличные от пуассоновского. Пуассоновское распределение гораздо чаще выступает в качестве предельного распределения для размерности пространства решений (см., например, [7-9]).

Напротив, предельное распределение Пуассона естественным образом возникает в задаче о числе пустых ячеек при случайном размещении частиц по ячейкам. 
Поэтому будет интересно интерпретировать полученные результаты с этой точки зрения.

Сопоставим уравнениям системы (1)-(2) множества

$$
B_{t}=\left(V^{n} \backslash\{0\}\right) \backslash \bar{B}_{t}
$$

где

$$
\bar{B}_{t}=\left\{x \in V^{n} \backslash\{0\}: f_{t}\left(l_{t}^{1}(x), \ldots, l_{t}^{1}(x)\right)=0\right\} .
$$

Множество $W$ будет множеством пустых ячеек при независимом размещении в ячейки множества $V^{n} \backslash\{0\}$ частиц комплектами $B_{1}, \ldots, B_{T}$. Напомним, что частицы $t$-го комплекта размещаются по одной во все ячейки множества $B_{t}, t=1, \ldots, T$.

Наиболее изученной в теории случайных размещений является схема, в которой комплекты (множества $B_{t}$ ) выбираются случайно и равновероятно из множеств всех $s_{t}$-подмножеств множества ячеек $\left(s_{1}, \ldots, s_{T}\right.$ - заданные детерминированные параметры). Такая схема называется равновероятной схемой размещения частиц комплектами. Пусть $\mu_{0}-$ число пустых ячеек в этой схеме размещения. Как показано в [10], необходимыми и достаточными для сходимости распределения величины $\mu_{0}$ к пуассоновскому распределению с параметром $\lambda$ являются условия

$$
\mathbf{E} \mu_{0} \rightarrow \lambda, \quad N-s_{T} \rightarrow \infty, \quad s_{1}+\ldots+s_{T-1} \rightarrow \infty
$$

(здесь считается, что $s_{1}, \ldots, s_{T-1} \leqslant s_{T}$; в нашем случае число ячеек $N=q^{n}-1$ ).

Таким образом, с точки зрения теории случайных размещений утверждение теоремы 2 представляется вполне естественным. Однако наш случай отличается от равновероятной схемы размещения частиц комплектами двумя особенностями. Вопервых, размеры комплектов в нашем случае являются случайными. Однако это обстоятельство при естественных предположениях не сказывается на асимптотических свойствах числа решений. Нетрудно проверить, что с вероятностью

$$
P_{t}=\left(q^{n}-1\right)^{-m_{t}} \prod_{k=0}^{m_{t}-1}\left(q^{n}-q^{k}\right) \geqslant 1-q^{m_{t}-n}
$$

линейные функции $l_{t}^{1}, \ldots, l_{t}^{m_{t}}$ линейно независимы. Пусть

$$
\delta_{t}=I\left\{0 \notin B\left(f_{t}\right)\right\}, \quad k_{t}=\left|B\left(f_{t}\right)\right| .
$$

Тогда

$$
\mathbf{P}\left\{\left|B_{t}\right|=q^{n}\left(1-k_{t} q^{-m_{t}}\right)-\delta_{t}\right\}=P_{t} .
$$

Учитывая приведенное неравенство для $P_{t}$, получаем, что

$$
\begin{aligned}
\mathbf{P}\left\{\left|B_{t}\right|\right. & \left.=q^{n}\left(1-k_{t} q^{-m_{t}}\right)-\delta_{t} \mid t=1, \ldots, T\right\} \\
& \geqslant \prod_{t=1}^{T}\left(1-q^{m_{t}-n}\right) \geqslant 1-q^{-n} \sum_{t=1}^{T} q^{m_{t}} .
\end{aligned}
$$

Значит, при условии

$$
q^{-n} \sum_{t=1}^{T} q^{m_{t}} \rightarrow 0
$$


схема размещения, отвечающая системе (1)-(2), эквивалентна (в смысле совпадения результатов размещения с вероятностью, стремящейся к единице) некоторой схеме с комплектами детерминированного размера.

Более важным обстоятельством является неравновероятность комплектов. В этом свойстве можно убедиться, взяв $q=2$ и функцию $f_{t}(z)=z \oplus 1$. Тогда носителем распределения случайного множества $B_{t}$ будет совокупность всех линейных подпространств пространства $V^{n}$. (из всех рассматриваемых пространств удален нулевой вектор). При условии $n-m_{t} \rightarrow \infty$ это распределение стягивается на совокупность $(n-1)$-мерных линейных подпространств (без нулевого вектора в каждом). Но последняя составляет лишь часть множества всех $\left(2^{n-1}-1\right)$-подмножеств множества $V^{n} \backslash\{0\}$. Значит, распределение случайного множества (комплекта) $B_{t}$ в этом случае существенно отличается от распределения комплектов в равновероятной схеме. Для других функций $f$ дело обстоит аналогично, но носитель распределения может иметь существенно более сложную структуру.

Перейдем к доказательствам.

Доказательство теоремы 1 во многом повторяет рассуждения из статей [1] и [2]. Поэтому мы приводим подробно лишь начальный фрагмент доказательства и те части, в которых имеются существенные отличия.

Система уравнений (1)-(2) эквивалентна системе из $T m_{t}$-мерных включений (здесь и далее под включением понимается соотношение типа вектор принадлежит множеству)

$$
\left(\begin{array}{lll}
a_{t 1}^{1} x_{1} \oplus & \ldots & \oplus a_{t n}^{1} x_{n} \\
a_{t 1}^{m_{t}} x_{1} \oplus & \ldots & \oplus a_{t n}^{m_{t}} x_{n}
\end{array}\right) \in B\left(f_{t}\right) .
$$

Запишем систему (10) из $T$ включений относительно вектора $x=\left(x_{1}, \ldots, x_{n}\right)$ (рассматриваемого далее как вектор-столбец) как одно включение

$$
A x \in B
$$

в пространстве размерности $M=m_{1}+\ldots+m_{T}$. Для этого обозначим матрицу размера $m_{t} \times n$ случайных коэффициентов линейных форм из левой части отдельного включения в (10) через $A_{t}$, а в качестве матрицы $A$ возьмем матрицу размера $M \times n$, полученную приписыванием матриц $A_{1}, \ldots, A_{T}$ друг под другом в естественном порядке. В качестве множества $B$ возьмем множество

$$
B=B\left(f_{1}\right) \times \ldots \times B\left(f_{T}\right) \subseteq V^{M} .
$$

Заметим, что

$$
|B|=\prod_{t=1}^{T}\left|B\left(f_{t}\right)\right|
$$

При выбранных матрице $A$ и множестве векторов $B$ система (10) запишется в виде (11).

Свойство коллинеарности индуцирует на множестве $V^{n} \backslash\left\{0^{n}\right\}$ ненулевых векторов отношение эквивалентности. Пусть $E_{1}, \ldots, E_{N}-$ классы эквивалентных (попарно коллинеарных) векторов. Тогда

$$
\nu=\sum_{k=1}^{N} I\left\{\bigcup_{x \in E_{k}} \bigcup_{b \in B} A x=b\right\}=\sum_{b \in B} \sum_{k=1}^{N} I\left\{\bigcup_{x \in E_{k}} A x=b\right\} .
$$


Сопоставим каждой паре значений $(x, b), x \in V^{n} \backslash\left\{0^{n}\right\}, b \in B$, случайную величину

$$
\eta_{x, b}=I\{A x=b\} \text {. }
$$

Тогда из (13) вытекает следующее выражение для факториальных моментов случайной величины $\nu$ :

$$
\mathbf{E}(\nu)_{r}=\sum_{S_{r}} \sum_{B^{r}} \mathbf{E} \eta_{x^{1}, b^{1}} \ldots \eta_{x^{r}, b^{r}}
$$

где

$$
S_{r}=\left\{\left(x^{1}, \ldots, x^{r}\right) \in\left(V^{n} \backslash\left\{0^{n}\right\}\right)^{r}: x^{i} \neq a x^{j}, i \neq j, a \in G F(q) \backslash\{0\}\right\} .
$$

Из равенств (12), (13) и (3) следует, что

$$
E \nu=\sum_{S_{1}, B} E \eta_{x^{1}, b^{1}}=|B|\left|V^{n} \backslash\left\{0^{n}\right\}\right| q^{-M}=\Lambda .
$$

Рассмотрим старшие факториальные моменты. Заметим, что слагаемые в (14), для которых

$$
a_{1} x^{i_{1}} \oplus \ldots \oplus a_{s} x^{i_{s}}=0^{n}, \quad a_{1} b^{i_{1}} \oplus \ldots \oplus a_{s} b^{i_{s}} \neq 0^{M},
$$

равны нулю. Отбросим такие слагаемые из суммы (14), а оставшиеся сліагаеые сгруппируем следующим образом. Каждому набору векторов $x^{1}, \ldots, x^{r} \in V^{n} \backslash\left\{0^{n}\right\}$ соответствует система $L_{x}\left(x^{1}, \ldots, x^{r}\right)$ однородных линейных уравнений в $V^{n}$ вида

$$
a_{1} x^{i_{1}} \oplus \ldots \oplus a_{s} x^{i_{s}}=0^{n}
$$

возможно пустая, состоящая из всех линейных соотношений, которым удовлетворяет набор векторов $x^{1}, \ldots, x^{r}$. Системе $L_{x}\left(x^{1}, \ldots, x^{r}\right)$ сопоставим идентичную по записи систему $L_{b}\left(x^{1}, \ldots, x^{r}\right)$ из линейных уравнений относительно $b^{1}, \ldots, b^{r} \in B$. Обозначим через $B\left(x^{1}, \ldots, x^{r}\right)$ множество решений $\left(b^{1}, \ldots, b^{r}\right) \in B^{r}$ системы уравнений $L_{b}\left(x^{1}, \ldots, x^{r}\right)$.

Введем обозначение

$$
S_{r, k}=\left\{\left(x^{1}, \ldots, x^{r}\right) \in S_{r}: \operatorname{rank}\left(x^{1}, \ldots, x^{r}\right)=k\right\} .
$$

Очевидно, что $S_{r, 0}=S_{r, 1}=\varnothing, r \geqslant 2$.

Тогда

$$
\mathbf{E}(\nu)_{r}=\sum_{k=2}^{r} \sum_{\left(x^{1}, \ldots, x^{r}\right) \in S_{r, k}} \sum_{B\left(x^{1}, \ldots, x^{r}\right)} E \eta_{x^{1}, b^{1}} \ldots \eta_{x^{r}, b^{r}} .
$$

Нетрудно убедиться в том, что если $\operatorname{rank}\left(x^{1}, \ldots, x^{r}\right)=r$, то

$$
B\left(x^{1}, \ldots, x^{r}\right)=B^{r}, \quad E \eta_{x^{1}, b^{1}} \ldots \eta_{x^{r}, b^{r}}=q^{-r M}
$$

Поэтому

$$
\sum_{\left(x^{1}, \ldots, x^{r}\right) \in S_{r, r}} \sum_{B\left(x^{1}, \ldots, x^{r}\right)} E \eta_{x^{1}, b^{1}} \ldots \eta_{x^{r}, b^{r}}=\frac{|B|^{r}\left|S_{r, r}\right|}{q^{r M}}
$$


С учетом равенства

$$
\left|S_{r, r}\right|=\prod_{s=0}^{r-1}\left(q^{n}-q^{s}\right)
$$

и формулы (12) из (16) и (17) выводим (4).

Из (16) и (17) вытекает также интересное равенство

$$
\mathrm{E}(\nu)_{2}=\frac{q^{n}-q}{q^{n}-1} \Lambda^{2}
$$

Перейдем к построению верхней оценки. Нетрудно проверить, что

$$
\mathrm{E} \eta_{x^{1}, b^{1}} \ldots \eta_{x^{r}, b^{r}} \leqslant q^{-k M}
$$

если $\operatorname{rank}\left(x^{1}, \ldots, x^{r}\right)=k$. Поэтому из (16) вытекает оценка при $n \geqslant r \geqslant 2$

$$
\mathbf{E}(\nu)_{r} \leqslant \sum_{k=2}^{r} \sum_{\left(x^{1}, \ldots, x^{r}\right) \in S_{r, k}} \frac{1}{q^{k M}}\left|B\left(x^{1}, \ldots, x^{r}\right)\right| .
$$

Обозначим через $C\left(r, d ; B_{1}, \ldots, B_{T}\right)$, где $d \in V^{M}$, число решений

$$
\left(u^{1}, \ldots, u^{r}\right), \quad u^{1}, \ldots, u^{r} \in B=B_{1} \times \ldots \times B_{T},
$$

уравнения

$$
a_{1} u^{1} \oplus \ldots \oplus a_{r} u^{r}=d
$$

где $a_{1}, \ldots, a_{r} \in G F(q) \backslash\{0\}$.

Лемма 1. Для любых $B_{1} \in V^{m_{1}}, \ldots, B_{T} \in V^{m_{T}} u d \in V^{M}$

$$
C\left(3, d ; B_{1}, \ldots, B_{T}\right) \leqslant|B|^{2} \prod_{t=1}^{T}\left(1-\chi\left(B_{t}\right)\left|B_{t}\right|^{-2}\right) .
$$

Следствие 1. Прu $r \geqslant 3 u \operatorname{любых~} B_{1} \in V^{m_{1}}, \ldots, B_{T} \in V^{m_{T}} u d \in V^{M}$

$$
C\left(r, d ; B_{1}, \ldots, B_{T}\right) \leqslant|B|^{r-1} \prod_{t=1}^{T}\left(1-\chi\left(B_{t}\right)\left|B_{t}\right|^{-2}\right) .
$$

При доказательстве леммы 1 понадобится следующее утверждение.

Лемма 2. Множество $B \subseteq V^{m}$ является $р$-аффинным тогда и только тогда, когда одно из множеств векторов

$$
b \oplus B=\{b \oplus z: z \in B\}, \quad b \in V^{m},
$$

является группой относителъно сложения в $V^{m}$.

Доказательство этого утверждения проводится стандартными рассуждениями, и мы его опускаем. 
Доказательство леммъ 1. Сначала рассмотрим случай $T=1$, в котором уравнение (20) (при $r=3$ )

$$
a_{1} u^{1} \oplus a_{2} u^{2} \oplus a_{3} u^{3}=d
$$

рассматривается над $B_{1}$. При этом, не нарушая общности, можно считать, что $a_{1}=1$. Очевидно, что $C\left(3, d ; B_{1}\right) \leqslant\left|B_{1}\right|^{2}$. Предположим, что

$$
C\left(3, d ; B_{1}\right)=\left|B_{1}\right|^{2} \text {. }
$$

В этом случае для любых $u^{1} \in B_{1} u^{2} \in B_{1}$ найдется $u^{3} \in B_{1}$, при котором

$$
d \ominus a_{3} b^{3}=b^{1} \oplus a_{2} b^{2}
$$

(мы используем запись $z=x \ominus y$, если $x=z \oplus y$ ). Это значит, что

$$
\left\{B_{1} \oplus a_{2} B_{1}\right\}=\left\{y \oplus a_{2} z: y, z \in B_{1}\right\} \subseteq d \ominus a_{3} B_{1},
$$

то есть $\left|\left\{B_{1} \oplus a_{2} B_{1}\right\}\right| \leqslant\left|B_{1}\right|$.

Если $0 \in B_{1}$, то $B_{1} \subseteq\left\{B_{1}+a_{2} B_{1}\right\}$. Следовательно, $\left|\left\{B_{1} \oplus a_{2} B_{1}\right\}\right|=\left|B_{1}\right|$ и

$$
\left\{B_{1} \oplus a_{2} B_{1}\right\}=B_{1} .
$$

Аналогично получаем, что $\left\{B_{1} \oplus a_{2} B_{1}\right\}=a_{2} B_{1}$. Значит, $a_{2} B_{1}=B_{1}$ и

$$
\left\{B_{1} \oplus B_{1}\right\}=\left\{B_{1} \oplus a_{2} B_{1}\right\}=B_{1} \text {. }
$$

Заметим теперь, что благодаря равенству $\left\{B_{1} \oplus B_{1}\right\}=B_{1}$ вместе с $b \in B_{1}$ в $B_{1}$ содержится элемент $-b=(p-1) b$. Поэтому множество $B_{1}$ является группой относительно покоординатного сложения.

Пусть $0 \notin B_{1}$. Тогда возьмем некоторый элемент $b \in B_{1}$ и рассмотрим множество $B^{\prime}=B_{1} \ominus b$. Уравнение (23) эквивалентно аналогичному уравнению вида (23) относительно $b^{1}, b^{2}, b^{3} \in B^{\prime}$ с $d^{\prime}=d \oplus b\left(b^{1} \oplus b^{2} \oplus b^{3}\right)$. Теперь $0 \in B^{\prime}$, и предшествующие рассуждения приводят к выводу, что множество $B_{1} \oplus(p-1) b=B_{1} \ominus b$ является группой. Поэтому, согласно лемме 2 , из (24) следует равенство $\chi\left(B_{1}\right)=0$.

Заметим теперь, что при нарушении равенства (24) выполнено соотношение

$$
C\left(3, d ; B_{1}\right) \leqslant\left|B_{1}\right|^{2}-1 \text {. }
$$

Все вместе это дает неравенство

$$
C\left(3, d ; B_{1}\right) \leqslant\left|B_{1}\right|^{2}\left(1-\chi\left(B_{1}\right)\left|B_{1}\right|^{-2}\right) .
$$

При $T \geqslant 2$ число решений над $B=B_{1} \times \ldots \times B_{T}$ равно произведению чисел решений над $B_{t}$. Отсюда получаем (21). Лемма 1 доказана.

Доказательство следствия 1. Зафиксируем в (20) неизвестные $b^{4}, \ldots, b^{r}$, что можно сделать не более, чем $|B|^{r-3}$ способами. При этом получаем уравнение вида (20) c $r=3$, число решений которого оценивается сверху с помощью неравенства (21). Отсюда и из (12) получаем оценку (22). Следствие доказано. 
Лемма 3. Пустъ $\operatorname{rank}\left(x^{1}, \ldots, x^{r}\right)=k u 2 \leqslant k \leqslant r-1$. Тогда

$$
\left|B\left(x^{1}, \ldots, x^{r}\right)\right| \leqslant|B|^{k} \prod_{t=1}^{T}\left(1-\chi\left(B_{t}\right)\left|B_{t}\right|^{-2}\right) .
$$

Доказательство. Перенумеруем векторы $x^{1}, \ldots, x^{r}$ так, чтобы первые $k$ из них составляли базис. Тогда систему $L_{b}\left(x^{1}, \ldots, x^{r}\right)$ можно преобразовать к виду

$$
\begin{aligned}
b^{k+1} & =l_{k+1}\left(b^{1}, \ldots, b^{k}\right), \\
& \cdots \\
b^{r} & =l_{r}\left(b^{1}, \ldots, b^{k}\right)
\end{aligned}
$$

с некоторыми линейными функциями $l_{k+1}, \ldots, l_{r}$ своих аргументов. Так как из этих уравнений значения $b^{k+2}, \ldots, b^{r} \in B$ определяются величинами $b^{1}, \ldots, b^{k}$ однозначно, число решений системы (26) относительно $b^{1}, \ldots, b^{r} \in B$ не превосходит числа решений уравнения $b^{k+1}=l_{k+1}\left(b^{1}, \ldots, b^{k}\right)$ относительно $b^{1}, \ldots, b^{k+1}$. Последнее с учетом неравенства (22) не превосходит

$$
|B|^{k} \prod_{t=1}^{T}\left(1-\chi\left(B_{t}\right)\left|B_{t}\right|^{-2}\right)
$$

если число $s$ существенных аргументов в выражении

$$
\left.b^{k+1} \ominus l_{k+1}\left(b^{1}, \ldots, b^{k}\right)\right)
$$

удовлетворяет неравенству $s \geqslant 3$. Это неравенство имеет место, поскольку в системах $L_{x}\left(x^{1}, \ldots, x^{r}\right)$ и $L_{b}\left(x^{1}, \ldots, x^{r}\right)$ нет одночленных и двучленных уравнений. Лемма 3 доказана.

Вернемся к неравенству (19). Применим оценку (25) к множествам $B_{t}=B\left(f_{t}\right)$ и подставим ее в (19) (для слагаемых с $k<r$ ). Воспользовавшись при этом равенствами (12) и (17), очевидными оценками

$$
\left|B\left(x^{1}, \ldots, x^{r}\right)\right|<|B|^{r}, \quad\left|S_{r, k}\right| \leqslant C_{r}^{k}\left(q^{k}-1\right)^{r-k} \prod_{s=0}^{k-1}\left(q^{n}-q^{s}\right),
$$

после несложных преобразований получим (5). Теорема 1 доказана.

Докажем теперь теорему 2. Заметим, что из условия (6) вытекает сходимость

$$
\prod_{t=1}^{T}\left(1-\chi\left(B\left(f_{t}\right)\right)\left|B\left(f_{t}\right)\right|^{-2}\right) \rightarrow 0 \text {. }
$$

Поэтому соотношение (7) следует из условия $\Lambda \rightarrow \lambda$ и неравенств (4), (5) теоремы 1. Из него, в свою очередь, следует сходимость распределения. Теорема 2 доказана.

Доказательство теоремы 3 аналогично доказательству теоремы 3 из [1], и мы его опускаем. 


\section{Список литературы}

1. Михайлов В. Г., Предельные теоремы для числа ненулевых решений одной системы случайных уравнений над полем $G F(2)$. Теория вероятностей и ее применения (1998) 43, №3, 598-606.

2. Михайлов В. Г., Предельные теоремы для числа ненулевых решений одной системы случайных уравнений над полем $G F(2)$. Дисхретная математиха (2000) 12, №1, 7081.

3. Коваленко И. Н., Левитская А. А., Савчук М. Н., Избранные задачи вероятностной комбинаторижи. Наукова Думка, Киев, 1986.

4. Колчин В. Ф., Системъ случайных уравнений. МИЭМ, Москва, 1988.

5. Балакин Г. В., Введение в теорию случайных систем уравнений. Труды по дискретной математике (1997) 1, 1-18.

6. Балакин Г. В., Системы случайных систем уравнений над конечным полем. Труды по дискретной математихе (1998) 2, 21-37.

7. Копытцев В. А., О распределении числа решений случайных заведомо совместных систем уравнений. Теория вероятностей и ее применения (1995) 40, №2, 430-437.

8. Михайлов В. Г., Предельные теоремы для случайного покрытия конечного множества и для числа решений системы случайных уравнений. Теория вероятностей и ее применения (1996) 41, №2, 272-283.

9. Копытцев В. А., Предельные теоремы для числа решений системы случайных уравнений. Теория вероятностей и ее применения (2000) 45, №1, 52-72.

10. Ватутин В. А., Михайлов В. Г., Предельные теоремы для числа пустых ячеек в равновероятной схеме размещения частиц комплектами. Теория вероятностей и ее применения (1982) 27, №4, 684-692.

Статья поступила 14.02.2001. 